\title{
Correction to: PCA-RECT: An Energy-Efficient Object Detection Approach for Event Cameras
}

Bharath Ramesh (D), Andrés Ussa, Luca Della Vedova, Hong Yang, and Garrick Orchard

\section{Correction to: \\ Chapter "PCA-RECT: An Energy-Efficient Object \\ Detection Approach for Event Cameras" \\ in: G. Carneiro and S. You (Eds.): \\ Computer Vision - ACCV 2018 Workshops, LNCS 11367, https://doi.org/10.1007/978-3-030-21074-8_35}

In the version of this chapter that was originally published, the funding information given at the bottom of the first page was not correct. This has been updated so that the new version now reads:

"Supported by Temasek Research Fellowship." 